\title{
A realização do sistema supersérie com diferentes segmentos corporais não impacta no volume e percepção subjetiva de esforço
}

\section{The superset system performed using different limbs does not impact on volume and rating of perceived exertion}

El sistema supersérie realizado con diferentes segmentos corporais no impacta en el volumen y percepción subjetiva del esfuerzo

(iD) Ricardo Reis Dinardi

Pontifícia Universidade Católica de Minas Gerais, Belo Horizonte, Minas Gerais, Brasil dinardi06@hotmail.com

(iD) Guilherme Augusto Coelho Oliveira Pontifícia Universidade Católica de Minas Gerais, Belo Horizonte, Minas Gerais, Brasil dinardi06@hotmail.com

(iD) Lucas Furtado de Barros

Pontifícia Universidade Católica de Minas Gerais, Belo Horizonte, Minas Gerais, Brasil dinardi06@hotmail.com

(iD) Rodrigo Cesar Ribeiro Diniz

Universidade Federal de Minas Gerais, Belo Horizonte, Minas Gerais, Brasil dinardi06@hotmail.com

iD José Mauro Silva Vidigal

Pontifícia Universidade Católica de Minas Gerais, Belo Horizonte, Minas Gerais, Brasil jmsvidigal30@gmail.com

iD Hugo Cesar Martins Costa

Pontifícia Universidade Católica de Minas Gerais, Belo Horizonte, Minas Gerais, Brasil hugopucminas@gmail.com 
Resumo: O objetivo do estudo foi comparar os sistemas tradicional (TRAD) e supersérie (SS) quanto ao número de repetições (volume) e percepção subjetiva de esforço (PSE) nos exercícios supino e extensão de joelhos. Doze homens treinados realizaram de forma randomizada (medidas repetidas) os protocolos em sistemas TRAD e SS (4 séries com pausas de 2 minutos, intensidade de 10 repetições máximas). No sistema SS, cada série do exercício banco extensor foi realizada durante a pausa do exercício supino. Não houve diferença significativa no volume (TRAD supino - série 1: $16,1 \pm 1,6$; série 2 : $10,9 \pm 2,1$; série 3: 7,8 $\pm 2,3$; série 4 : 7,0 \pm 2,0; TRAD extensão de joelhos - série 1: 13,1 $\pm 2,5$; série 2: 11,0 \pm 2,0; série 3: $9,1 \pm 1,7$; série 4: 7,6 $\pm 1,6$; SS supino - série 1: $16,3 \pm 2,3$; série 2: 10,9 $\pm 1,6$; série 3: 7,8 $\pm 1,9$; série 4: 6,1 $\pm 1,4$; SS extensão de joelhos - série 1: $13,8 \pm 1,8$; série 2 : $11,2 \pm 1,6$; série $3: 8,8 \pm 1,7$; série 4: 7,0 $\pm 1,7$ ) e na PSE (TRAD supino - mediana série 1: 15,0; mediana série 4: 19,5; TRAD extensão de joelhos - mediana série 1: 17,0; mediana série 4: 19,5; SS supino - mediana série 1: 16,5; mediana série 4: 20,0; SS extensão de joelhos - mediana série 1: 17,5; mediana série 4: 19,5) entre os protocolos. Verificou-se redução do volume e aumento da PSE no decorrer das séries em ambas situações experimentais. Considerando a similaridade dos resultados obtidos entre os sistemas SS e TRAD, a aplicação de SS torna-se uma alternativa eficiente para a redução do tempo de treinamento.

Palavras-chave: Exercício. Aptidão Física. Musculação.

Abstract: The objective of the study was to compare the number of repetitions (volume) and the rating of perceived exertion (RPE) in the bench press and knee extension exercises when performing Traditional (TRAD) and Superset (SS) training systems. Twelve trained men randomly performed (repeated measurements) the TRAD and SS systems (4 sets and 2-minute rest intervals, intensity of 10 max repetitions). In the SS system, each set of the knee extension was performed during the interval of the bench press. There was no significant difference in volume (TRAD bench press - set 1: $16,1 \pm 1,6$; set $2: 10,9 \pm 2,1$; set $3: 7,8 \pm 2,3$; set 4: 7,0 $\pm 2,0$; TRAD knee extension - set 1: $13,1 \pm 2,5$; set 2: $11,0 \pm 2,0$; set 3: 9,1 $\pm 1,7$; set 4: 7,6 $\pm 1,6$; SS bench press - set 1: $16,3 \pm 2,3$; set 2: $10,9 \pm 1,6$; set 3: 7,8 $\pm 1,9$; set $4: 6,1 \pm 1,4$; SS knee extension - set 1: $13,8 \pm 1,8$; set 
2: $11,2 \pm 1,6$; set 3: $8,8 \pm 1,7$; set $4: 7,0 \pm 1,7$ ) and PSE (TRAD bench press median set 1: 15,0; median set 2: 19,5; TRAD knee extension - median set 1: 17,0; median set 4: 19,5; SS bench press - median set 1:16,5; median set 4: 20,0; SS knee extension - median set 1: 17,5; median set 4: 19,5) between the protocols. There was a reduction in volume and increase of PSE during the sets in both experimental situations. Considering the similarity of the results obtained between the SS and TRAD systems, SS application becomes an efficient alternative for decreasing training time.

Keywords: Exercise. Physical Fitness. Resistance Training.

Resumen: El objetivo del estudio fue comparar los sistemas Tradicional (TRAD) y Supersérie (SS) en cuanto al número de repeticiones (volumen) y percepción subjetiva de esfuerzo (PSE) en los ejercicios press de banca (PB) y extensión de rodillas (ER). Doce hombres entrenados realizaron de forma aleatorizada (mediciones repetidas) los sistemas TRAD y SS (4 series; pausas de 2 minutos, intensidad de 10 repeticiones máximas). En el sistema SS, cada serie de ER se realizó durante la pausa de PB. No hubo diferencia en el volumen (TRAD press de banca - serie 1: 16,1 \pm 1,6; serie 2: 10,9 $\pm 2,1$; serie 3: 7,8 $\pm 2,3$; serie 4: 7,0 $\pm 2,0$; TRAD extensión de rodillas - serie 1: $13,1 \pm 2,5$; serie 2 : $11,0 \pm 2,0$; serie $3: \quad 9,1 \pm 1,7$; serie 4: 7,6 $\pm 1,6$; SS press de banca - serie 1: 16,3 $\pm 2,3$; serie 2: 10,9 \pm 1,6; serie $3: 7,8 \pm 1,9$; serie $4: 6,1 \pm 1,4 ;$ SS extensión de rodillas - serie 1: $13,8 \pm 1,8$; serie $2: 11,2 \pm 1,6$; serie $3: 8,8 \pm 1,7$; serie $4: 7,0 \pm 1,7$ ) e na PSE (TRAD press de banca - mediana serie 1: 15,0; mediana serie 4: 19,5; TRAD extensión de rodillas - mediana serie 1: 17,0; mediana serie 4: 19,5; SS press de banca - mediana serie 1: 16,5; mediana serie 4: 20,0; SS extensión de rodillas - mediana serie 1: 17,5; mediana serie 4: 19,5) entre los protocolos. Se verificó reducción del volumen y aumento de la PSE en el curso de las series en ambas situaciones. Considerando la similitud de los resultados obtenidos entre los sistemas SS y TRAD, la aplicación de SS se convierte en una alternativa eficiente para la reducción del tiempo de entrenamiento.

Palabras-clave: Ejercicio. Aptitud Física. Entrenamiento de Resistencia.

Submetido em: 25/06/2018

Aceito em: 17/12/2019 
A realização do sistema supersérie com diferentes segmentos corporais... Ricardo Reis Dinardi • Guilherme Augusto Coelho Oliveira, et al...

\section{Introdução}

A prescrição do treinamento de força na musculação é realizada pela manipulação de diferentes variáveis, tais como número de repetições, duração da repetição, pausa, carga (ACSM, 2009; BIRD et al., 2005; KRAEMMER; RATAMESS, 2004), sendo que diferentes possibilidades de organização dessas variáveis permitem a estruturação dos chamados "sistemas de treinamento" (FLECK; KRAEMMER, 2017).

Dentre os diferentes sistemas de treinamento citados na literatura (FLECK; KRAEMMER, 2017), as superséries (SS) são caracterizadas genericamente pela realização de dois exercícios desempenhados de forma alternada, sem pausa entre eles, sendo tal execução realizada por grupos musculares diferentes (ROBBINS et al., 2010a; WEAKLEY et al., 2017a; WEAKLEY et al., 2017b). Enquanto que em protocolos de treinamento tradicionais (TRAD), o praticante realiza normalmente séries múltiplas de um exercício (ex: extensão de joelhos) seguido de outro exercício (ex: flexão de joelhos); no protocolo em SS, por sua vez, o sujeito realizaria o segundo exercício imediatamente após ou durante período de pausa entre séries do primeiro exercício (ex: flexão de joelhos realizada na pausa da extensão de joelhos). Uma das importantes implicações da realização das SS seria a redução do tempo total de treinamento (BALSAMO et al., 2012; PAZ et al., 2014; ROBBINS et al., 2009), o que impactaria positivamente na rotina de treinamento de atletas (WEAKLEY et al., 2017a; WEAKLEY et al., 2017b) ou mesmo de não atletas (PAZ et al., 2014). Destaca-se que a "falta de tempo" tem sido indicada como um dos principais fatores associados à desistência da prática da musculação em academias de ginástica (LIZ; ANDRADE, 2016).

Na última década tem crescido o número de investigações visando a elucidar o efeito da utilização das SS (BALSAMO et al., 2012; CORRÊA et al., 2017; ROBBINS et al., 2009; SOUZA et al., 2017; 
A realização do sistema supersérie com diferentes segmentos corporais... Ricardo Reis Dinardi • Guilherme Augusto Coelho Oliveira, et al...

WEAKLEY et al., 2017a; WEAKLEY et al., 2017b). Parte dos estudos envolvendo o efeito agudo do sistema SS tem se centrado no impacto provocado no volume de treinamento, normalmente definido pelo número de repetições executadas na sessão de treinamento (repetições x séries) ou mesmo o produto entre o número de repetições e o peso levantado pelo praticante (repetições $x$ séries $\times$ kg) (BALSAMO et al., 2012; MONTEIRO et al., 2015; SENNA et al., 2010). Dentre outros motivos, o interesse no volume de treinamento se justifica pela sua associação com ganhos de força (RHEA et al., 2003; SCHOENFELD et al., 2017; SUCHOMEL et al., 2018) e hipertrofia muscular (SUCHOMEL et al., 2018) em programas de musculação.

Embora pesquisas relacionadas ao efeito das SS no volume de treinamento têm sido aplicadas aos exercícios agonistas-antagonistas pareados (BALSAMO et al., 2012; PAZ et al., 2014; ROBBINS et al., 2009; SENNA et al., 2010), outras formas de organização das SS também são analisadas, como, por exemplo, alternar exercícios de segmentos corporais diferentes (membros inferiores $x$ membros superiores) (MONTEIRO et al., 2015; NASSER et al., 2019; WEAKLEY et al., 2017a; WEAKLEY et al., 2017b). Entretanto, tal tipo de organização das SS tem sido menos explorado nos estudos. Senna et al. (2010), por exemplo, compararam protocolos TRAD e SS no volume total de treinamento (VTT). Os autores analisaram uma única série de dois exercícios (supino horizontal e extensão de joelhos), e foi verificado que a realização do SS não impactou no volume total de treinamento. Deve-se destacar, contudo, que essa pesquisa não utilizou séries múltiplas, organização de treinamento preconizada em protocolos na musculação (ACSM, 2009; BIRD et al., 2005).

Considerando que protocolos realizados com séries múltiplas promovem maior resposta metabólica (MÁRQUEZ et al., 2017; SUCHOMEL et al., 2018) e que o aumento da concentração de lactato e íons $\mathrm{H}+$ parece estar associado à redução de produção de força em exercícios alternados por segmentos (MÁRQUEZ et al., 2017), é possível que a realização de séries múltiplas em protocolos de SS executadas com segmentos corporais diferentes possa im- 
A realização do sistema supersérie com diferentes segmentos corporais... Ricardo Reis Dinardi • Guilherme Augusto Coelho Oliveira, et al...

pactar na redução do VTT quando comparado a protocolos TRAD. Recentemente, Monteiro et al. (2015) confirmaram essa hipótese. Foram comparados protocolos TRAD e protocolos SS realizados de forma alternada por segmentos no VTT. Os autores verificaram redução do VTT ao realizar o protocolo alternado por segmentos, indicando um impacto negativo no desempenho ao se realizar as SS dessa forma. Entretanto, destaca-se que a pausa utilizada entre séries no estudo de Monteiro et al. (2015) foi menor na condição SS quando comparado ao TRAD, fator que pode também impactar no volume de treinamento (WILLARDSON, 2006). Outro estudo também realizado recentemente comparou protocolos TRAD e SS de forma alternada por segmento (WEAKLEY et al., 2017a) com séries múltiplas. Os autores verificaram maior eficiência de treinamento (VTT dividido pela duração do treinamento) ao se executar o protocolo SS em comparação ao TRAD. Entretanto, nesse estudo, não foram comparados parâmetros de volume nas condições experimentais citadas (WEAKLEY et al., 2017a).

A percepção subjetiva de esforço (PSE) tem demonstrado ser um parâmetro interno sensível a alterações da carga de treinamento na musculação (TIGGEMANN; KRUEL, 2010). Diferentes estratégias têm sido adotadas ao se avaliar PSE em protocolos de treinamento de força (NACLERIO et al., 2015), como o registro geral da sessão de treinamento (WEAKLEY et al., 2017b) ou dos músculos utilizados especificamente na tarefa (DINIZ et al., 2014; HISCOCK et al., 2016). Particularmente em protocolos realizados em SS, há indicativos de que a utilização desse sistema de treinamento possa aumentar a PSE da sessão de treinamento (WEAKLEY et al., 2017b). Entretanto, não foram encontrados estudos que comparassem a PSE dos músculos utilizados durante protocolos em SS e TRAD. Considerando que a resposta da PSE dos músculos exercitados parece ser afetada pelo VTT (HISCOCK et al., 2016), a expectativa da redução desse componente durante a realização do treinamento em SS possivelmente impactará na PSE dos praticantes.

Com base no exposto, não está claro qual é o impacto da realização de protocolos SS utilizando séries múltiplas no volume de 
A realização do sistema supersérie com diferentes segmentos corporais... Ricardo Reis Dinardi • Guilherme Augusto Coelho Oliveira, et al...

treinamento e percepção de esforço do praticante, especialmente quando a comparação é realizada com protocolos TRAD utilizando equiparação de pausas entre séries. Portanto, o objetivo do presente estudo é avaliar o efeito agudo do protocolo SS (alternado por segmento) sobre o número de repetições e PSE quando comparado ao protocolo TRAD na musculação.

\section{Métodos}

\section{Amostra}

Participaram do estudo 12 voluntários do sexo masculino (idade média 23,5 $\pm 4,2$ anos; massa corporal 72,6 $\pm 7,6 \mathrm{~kg}$; estatura $176,7 \pm 4,9 \mathrm{~cm})$. Como critério de inclusão, os voluntários deveriam ter experiência mínima de 6 meses contínuos de treinamento de força na musculação (ACSM, 2009), bem como não apresentar limitações funcionais para a realização dos exercícios utilizados no presente estudo (supino e extensão de joelhos). Além disso, foi solicitado que os voluntários alterassem suas rotinas de treinamento durante o período de coleta de dados, a fim de evitar a realização de exercícios que utilizassem o músculo peitoral maior, tríceps braquial, deltóide anterior e quadríceps $48 \mathrm{~h}$ antes das sessões de coleta.

\section{Questões éticas}

Todos os participantes foram informados sobre os procedimentos e objetivos da pesquisa e assinaram o Termo de Consentimento Livre e Esclarecido (TCLE). O presente estudo foi aprovado pela universidade na qual a pesquisa foi realizada (CAAE: 58961616.0.0000.5137). 
A realização do sistema supersérie com diferentes segmentos corporais... Ricardo Reis Dinardi • Guilherme Augusto Coelho Oliveira, et al...

\section{Desenho experimental}

O presente estudo utilizou um delineamento experimental de medidas repetidas para analisar o efeito dos protocolos de treinamento TRAD e SS no volume de treinamento (número total de repetições) e PSE. Cada voluntário compareceu ao local de coleta de dados em 4 dias diferentes - sessões experimentais 1 a 4 -, separadas por pelo menos 2 e no máximo de 7 dias. A ordem de realização dos protocolos por cada voluntário ocorreu de forma randomizada (Figura 1).

Figura 1. Desenho do estudo experimental do estudo.

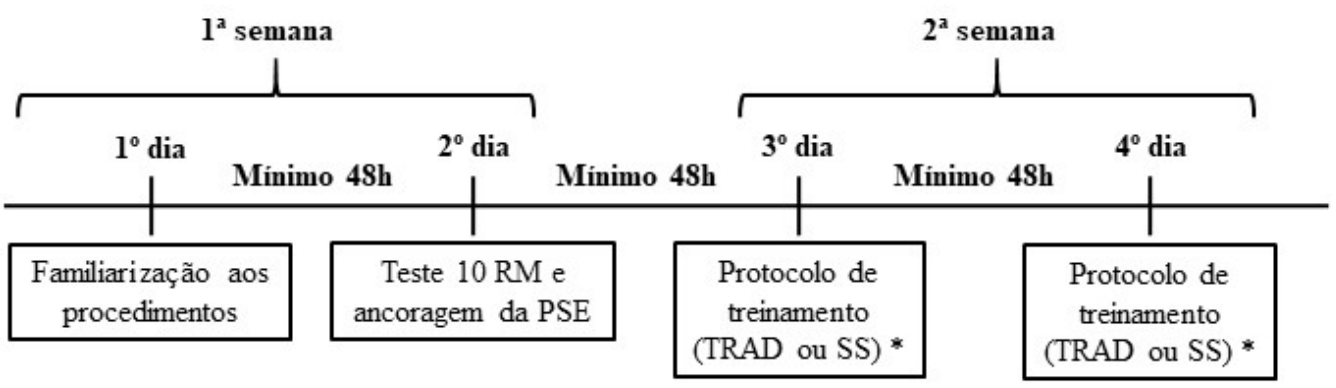

Legenda: 10 RM: teste de 10 repetições máximas; PSE: percepção subjetiva de esforço; TRAD: Protocolo de treinamento Tradicional; SS: Protocolo de Treinamento Supersérie; * realização balanceada entre os protocolos. Fonte: Elaboração própria.

\section{Sessões experimentais 1 e 2}

Na sessão experimental 1, depois de realizadas as medidas antropométricas, os voluntários foram submetidos à familiarização ao teste de 10 repetições máximas (10RM) nos exercícios supino reto e extensão de joelhos, respectivamente nessa ordem. Para o exercício supino, foi solicitado que o voluntário segurasse a barra (10 kg) de maneira mais próxima a sua rotina de treinamento e realizasse cinco repetições sem a adição de anilhas para que fosse demarcado o posicionamento das mãos na barra. Nesse exercício, a barra deveria ser retirada do suporte com a ajuda dos pesquisadores até que o voluntário estendesse totalmente os co- 
A realização do sistema supersérie com diferentes segmentos corporais... Ricardo Reis Dinardi • Guilherme Augusto Coelho Oliveira, et al...

tovelos, sendo este definido como limite superior. O limite inferior da amplitude de movimento ocorria quando a barra tocava em um retângulo de EVA $(20 \mathrm{~cm} \times 10 \mathrm{~cm})$ posicionado sobre o osso esterno do indivíduo.

Para a realização do exercício de membros inferiores, foi utilizado um equipamento banco extensor (Impulse, São Paulo, Brasil). Após ajustar a distância individualizada do encosto, o voluntário iniciava o movimento com um ângulo articular de $90^{\circ}$ de flexão de joelhos $\left(0^{\circ}=\right.$ extensão completa dos joelhos). Uma faixa elástica foi posicionada no equipamento de forma que os sujeitos devessem atingir, no mínimo, um ângulo de $10^{\circ}$ durante a ação concêntrica (extensão dos joelhos). Esse parâmetro visual foi utilizado para definir a amplitude de movimento final e consequentemente validar a execução de qualquer tentativa, seja do teste de 10RM ou da execução dos protocolos de treinamento.

\section{Teste de 10 repetições máximas (10RM)}

Na sessão experimental 2, os voluntários foram submetidos ao teste de 10RM (MAIA et al., 2014). Assim como no dia da familiarização, todos iniciaram pelo exercício supino, seguindo do exercício de extensão de joelhos. Pausas de 4 a 5 minutos foram dadas entre as séries e 5 minutos entre os exercícios. Foram necessárias entre 3 a 4 tentativas para encontrar o valor de 10RM em cada um dos exercícios, sendo o ajuste de peso mínimo correspondente a 2 kg em ambos os exercícios.

\section{Percepção subjetiva do esforço (PSE)}

Ainda na sessão experimental 2, foram também determinados os limites individuais de referência para o uso da PSE (GEARHART et al., 2001). Foi utilizada a escala de 15 níveis (BORG, 2000), iniciando do valor 6 e tendo como último ponto o valor 20. No presente estudo, os valores da escala de PSE foram relacionados à 
A realização do sistema supersérie com diferentes segmentos corporais... Ricardo Reis Dinardi • Guilherme Augusto Coelho Oliveira, et al...

percepção de esforço dos músculos ativos nos exercícios executados. Instruções padronizadas, adaptadas do estudo de Gearhart et al. (2001) foram utilizadas nesse estudo. Determinou-se os limites inferiores e superiores da escala PSE, sendo o valor 7 associado à sensação da realização de uma única repetição com o peso mínimo nos exercícios de extensão de joelhos e supino (7 kg e 10 $\mathrm{kg}$, respectivamente), e valor 19 , associado à sensação da última repetição executada em 10RM de cada um dos exercícios citados.

\section{Sessões experimentais 3 e 4}

Nas sessões 3 e 4 foram realizados os protocolos TRAD e SS de forma aleatória e balanceada entre os voluntários (Figura 2). 0 peso utilizado nos protocolos correspondeu a 90\% do desempenho de 10RM obtido na sessão experimental 2, sendo registrado o número máximo de repetições em cada série em cada um dos exercícios.

\section{Protocolo tradicional (TRAD)}

O protocolo TRAD consistiu na execução de 4 séries do exercício supino, seguido de 4 séries do exercício de extensão de joeIhos. Foram adotadas pausas de 2 minutos entre séries e 3 minutos entre exercícios.

\section{Protocolo supersérie (SS)}

No protocolo SS, 30 segundos após o término de cada série do exercício supino, o voluntário realizava o exercício de extensão de joelhos. Depois de finalizado este último, o voluntário aguardava o término da pausa do exercício supino (2 minutos) para reiniciar o primeiro exercício. A duração da repetição foi livre. A orientação dada aos voluntários foi que se aproximassem da rotina em que treinavam. Portanto, considerando que a duração da série 
A realização do sistema supersérie com diferentes segmentos corporais... Ricardo Reis Dinardi • Guilherme Augusto Coelho Oliveira, et al...

do segundo exercício (extensão de joelhos) foi sempre inferior a 90 segundos (isto é, o tempo necessário para reiniciar o exercício supino), os intervalos entre as séries foram mantidos de forma semelhante entre os dois protocolos. Os exercícios seriam interrompidos quando ocorressem falha concêntrica ou se as amplitudes propostas não fossem alcançadas. Os valores da PSE foram coletados após a $1^{\mathrm{a}}$ e $4^{\mathrm{a}}$ séries de cada exercício.

Figura 2. Representação esquemática dos protocolos supersérie (A) e tradicional (B)
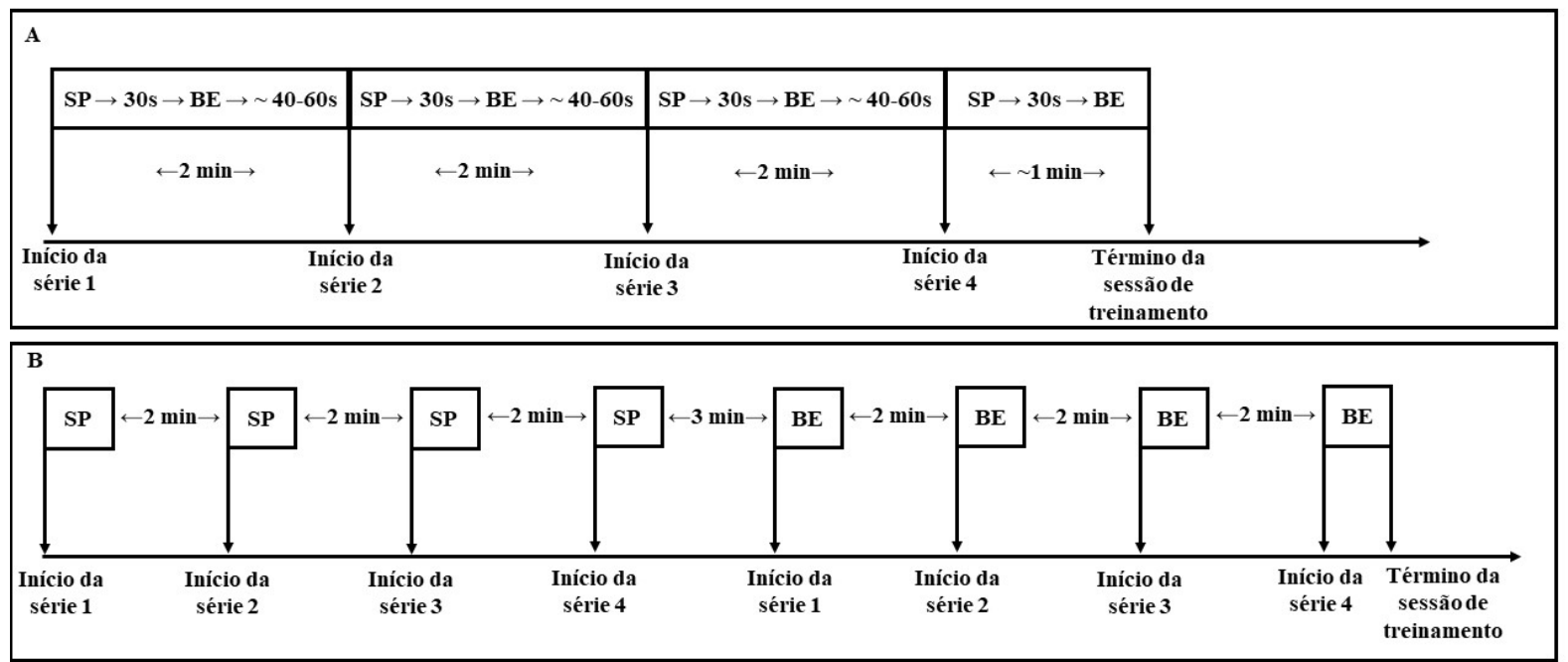

Legenda: SP: Supino; BE: Banco extensor. Fonte: Elaboração própria.

\section{Análise estatística}

Foi verificada a normalidade dos dados e homogeneidade das variâncias (testes de Shapiro-Wilk e Levene, respectivamente). Como a resposta da PSE não apresentou distribuição normal, os dados dessa variável foram apresentados por mediana, $1^{\circ}$ e $3^{\circ}$ quartis. Os demais dados foram representados em média e desvio-padrão. As comparações do número de repetições em cada exercício (supino e extensão de joelhos) foram feitas por meio da Análise de Variância (ANOVA) com medidas repetidas, tendo dois fatores (Protocolo de treinamento e Séries), seguida pelo post 
A realização do sistema supersérie com diferentes segmentos corporais... Ricardo Reis Dinardi • Guilherme Augusto Coelho Oliveira, et al...

hoc de Bonferroni para identificação das diferenças significativas, quando necessário. As comparações de PSE foram feitas por meio de teste de Friedman. O teste de Dunn foi utilizado como post hoc nesta situação. Assumiu-se o valor de $p<0,05$ para as análises. Os procedimentos estatísticos foram realizados no software SPSS 22.0.

\section{Resultados}

A figura 3 apresenta a comparação do número de repetições entre os protocolos no exercício supino e extensão de joelhos. A partir da ANOVA, verificou-se no supino efeito principal do fator série $\left(F_{3,33}=461,8 ; p<0,001\right)$, porém não foi verificado efeito principal do fator protocolo $\left(F_{1,11}=1,3 ; p=0,133\right)$ ou de interação $\left(F_{3,33}=2,1\right.$; $p=0,117)$. A análise de post hoc demonstrou redução do número de repetições ao longo das três primeiras séries $(p=0,001)$, entretanto não ocorreram diferenças da $3^{a}$ para a $4^{a}$ série $(p=0,139)$.

A partir da ANOVA, no exercício extensão de joelhos, verificou-se efeito principal do fator série $\left(F_{3,33}=171,2 ; p<0,001\right)$, porém não foi verificado efeito principal do fator protocolo $\left(F_{1,11}=0,10 ; p=\right.$ $0,958)$. Também não foi identificada efeito de interação $\left(F_{3,33}=2,3\right.$; $p=0,100)$. A análise de post hoc demonstrou redução do número de repetições ao longo de todas as séries $\left(1^{\mathrm{a}}>2^{\mathrm{a}} ; \mathrm{p}<0,007,2^{\mathrm{a}}>3^{\mathrm{a}}\right.$; $p=0,001,3^{a}>4^{a} ; p=0,013$. 
A realização do sistema supersérie com diferentes segmentos corporais... Ricardo Reis Dinardi • Guilherme Augusto Coelho Oliveira, et al...

Figura 3: média e desvio padrão do desempenho do número de repetições no exercício supino e extensão de joelhos durante os sistemas Tradicional e Supersérie $(n=12)$.

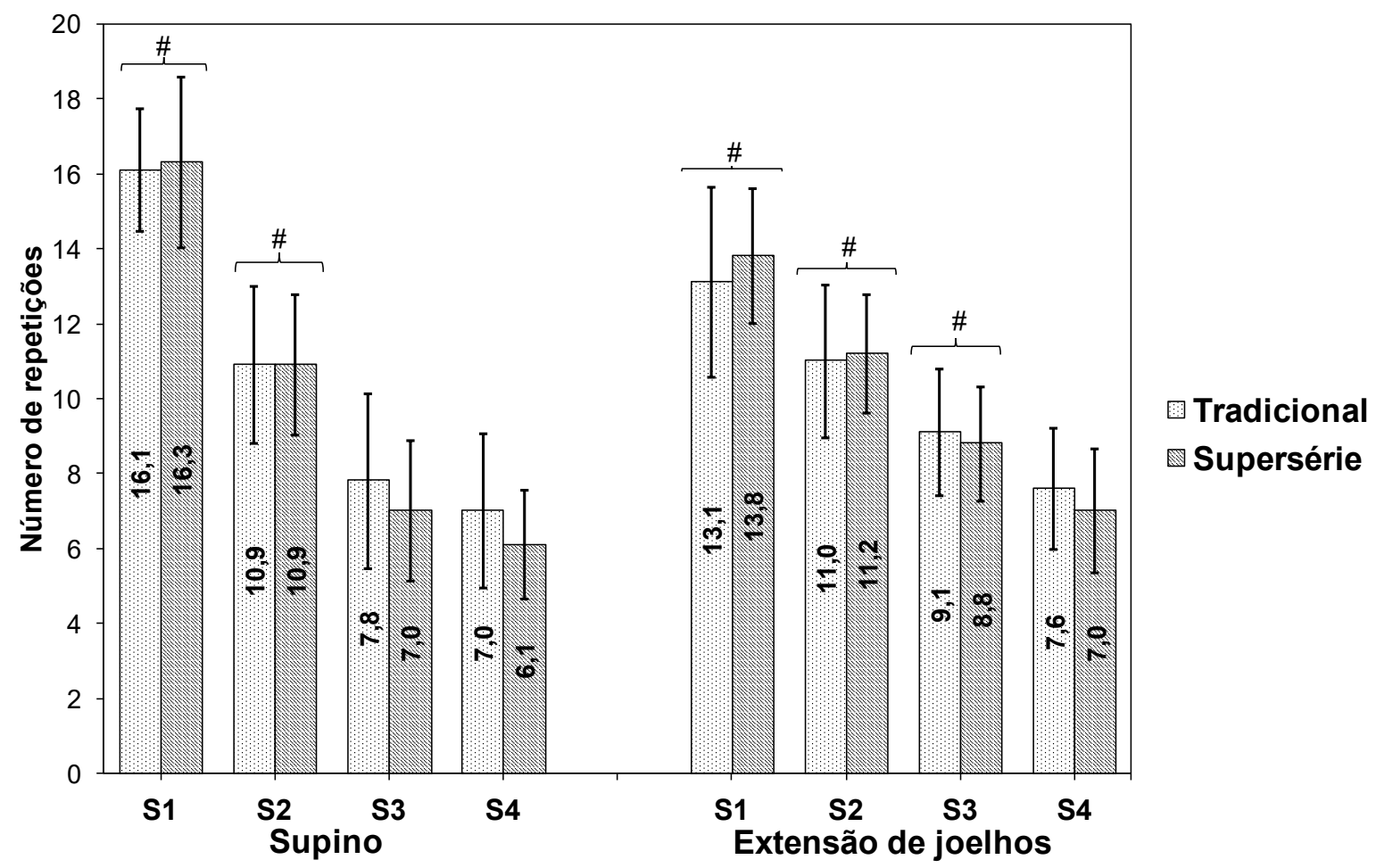

\#Número de repetições maior que na série subsequente. Fonte: Elaboração própria.

Os dados referentes à PSE para o exercício supino e extensão de joelhos encontram-se na Tabela 1. Houve diferenças na PSE tanto para o exercício supino $\left(\chi^{2}=25,6 ; p<0,001\right)$ quanto no exercício extensão de joelhos $\left(\chi^{2}=21,7 ; p<0,001\right)$. Na análise post hoc para o exercício supino, verificou-se aumento da PSE tanto no protocolo TRAD $(p=0,001)$ quanto no protocolo SS $(p=0,021) d a$ $1^{\text {a }}$ série para a $4^{\mathrm{a}}$ série, mas sem diferenças entre protocolos, na $1^{\text {a }}$ série $(p>0,99)$ e na $4^{a}$ série $(p>0,99)$. Resultados similares foram verificados na análise post hoc para o exercício extensão de joeIhos. Foi demonstrado aumento da PSE ao longo das séries tanto no protocolo TRAD $(p=0,027)$ quanto no protocolo SS $(p=0,007)$, mas sem diferenças entre protocolos, seja na $1^{\text {a }}$ série $(p>0,99)$ ou na $4^{\text {a }}$ série $(p>0,99)$. 
A realização do sistema supersérie com diferentes segmentos corporais...

Ricardo Reis Dinardi • Guilherme Augusto Coelho Oliveira, et al...

Tabela 1: Valores descritivos de PSE para o protocolo Tradicional e Supersérie $(n=12)$.

\begin{tabular}{|c|c|c|c|c|}
\hline \multirow[b]{2}{*}{ Protocolo } & \multirow[b]{2}{*}{ Série } & \multirow{2}{*}{$\begin{array}{l}\text { Medidas } \\
\text { Descritivas }\end{array}$} & \multicolumn{2}{|c|}{ Exercício } \\
\hline & & & Supino & $\begin{array}{c}\text { Extensão de } \\
\text { joelhos }\end{array}$ \\
\hline \multirow[t]{6}{*}{ Tradicional } & 1* & Q1 & 14,3 & 15,3 \\
\hline & & Mediana & 15,0 & 17,0 \\
\hline & & Q3 & 16,8 & 18,8 \\
\hline & 4 & Q1 & 18,3 & 18,3 \\
\hline & & Mediana & 19,5 & 19,5 \\
\hline & & Q3 & 20,0 & 20,0 \\
\hline \multirow[t]{6}{*}{ Supersérie } & $1 *$ & Q1 & 13,5 & 13,8 \\
\hline & & Mediana & 16,5 & 17,0 \\
\hline & & Q3 & 18,5 & 18,8 \\
\hline & 4 & Q1 & 19,0 & 19,0 \\
\hline & & Mediana & 20,0 & 19,5 \\
\hline & & Q3 & 20,0 & 20,0 \\
\hline
\end{tabular}

Nota: $Q_{1}=$ primeiro quartil, $Q_{3}=$ terceiro quartil. *Diferença significativa em relação à $4^{a}$ série: $p<0,05$. Fonte: Elaboração própria.

\section{Discussão}

O principal achado do presente estudo foi que os protocolos TRAD e SS executados por indivíduos treinados em musculação não apresentaram diferenças significativas no VTT e PSE. Adicionalmente, foi verificado uma redução do VTT e um aumento da PSE ao longo das séries, independentemente do protocolo adotado.

Conforme previamente apresentado, várias pesquisas compararam o efeito agudo do SS e TRAD no volume de treinamento, embora apenas alguns desses estudos utilizaram especificamente SS de forma alternada por segmento corporal (MONTEIRO et al., 2015; NASSER et al., 2019; SENNA et al., 2010). Desses estudos, a maior parte estruturou os protocolos em séries múltiplas, apresentando resultados divergentes. Monteiro et al. (2015) compara- 
A realização do sistema supersérie com diferentes segmentos corporais... Ricardo Reis Dinardi • Guilherme Augusto Coelho Oliveira, et al...

ram o número de repetições executadas em protocolos TRAD e SS nos exercícios supino e leg press (quatro séries, 10RM e pausas de 3 minutos). Os autores verificaram redução do volume ao realizar o protocolo em SS. Recentemente, Nasser et al. (2019) submeteram também sujeitos com experiência em musculação aos métodos TRAD e SS. Foram realizadas três séries com intensidade de 15RM (flexão de cotovelo, flexão de joelhos, extensão de cotovelos, extensão de joelhos), sendo adotadas pausas de um minuto tanto entre séries (protocolo TRAD) quanto entre pares de exercícios (protocolo SS). Os autores concluíram que o VTT foi maior na condição SS quando comparado com a TRAD.

No presente estudo, não ocorreram diferenças no volume de treinamento. Entretanto, o desenho experimental proposto procurou-se aproximar o tempo de pausa entre séries de cada exercício (2 minutos), independentemente do sistema de treinamento adotado, já que no protocolo SS a pausa era iniciada após o término do primeiro exercício (supino). Considerando que a pausa impacta na recuperação das reservas energéticas e consequentemente no desempenho agudo na musculação (WILLARDSON, 2006), é possível que a aparente contradição apresentada nos estudos de Monteiro (2015) e Nasser (2019) possa estar relacionada às diferentes pausas entre séries dos exercícios nos protocolos TRAD e SS. Assim como no presente estudo, Alcaraz et al. (2008) também quantificaram o tempo de pausa a partir do término do primeiro exercício em um sistema de Circuito (sequência de três exercícios), promovendo uma aproximação do tempo de intervalo entre séries dos diferentes exercícios executados pelos voluntários. Nesta pesquisa, também não foram verificadas diferenças no volume de treinamento do protocolo Circuito ao ser comparado com o protocolo TRAD. Deve-se salientar, entretanto, que o número de exercícios e a carga de treinamento adotada no estudo de Alcaraz et al. (2018) (três séries de 6RM, com pausas de três minutos entre séries) foram diferentes em relação ao adotado aqui. Portanto, as comparações devem ser feitas com cautela. 
A realização do sistema supersérie com diferentes segmentos corporais... Ricardo Reis Dinardi • Guilherme Augusto Coelho Oliveira, et al...

Outro dado que pode ser ressaltado é a melhoria da eficiência da sessão de treinamento obtida no sistema SS (NASSER et al., 2019; PAZ et al. 2014; ROBBINS et al. 2009; WEAKLEY et al. 2017a). Weakley et al. (2017a), por exemplo, compararam os protocolos TRAD e SS em sujeitos treinados em musculação. Os autores mencionaram uma melhor eficiência de treinamento (kg levantado por minuto) no protocolo SS. Embora, no presente estudo, a duração total da sessão não tenha sido quantificada com precisão, o tempo de treinamento realizado pelo protocolo SS foi consideravelmente menor que o TRAD (Figura 2). Nesse sentido, pode-se também assumir aqui uma maior eficiência do método SS.

No presente estudo verificou-se um aumento da PSE ao longo das séries. Esse resultado corrobora o estudo conduzido por Souza et al. (2017). Dez homens treinados em musculação foram submetidos três séries dos protocolos TRAD e SS (agonista-antagonista), utilizando os exercícios remada e supino, sendo também observado aumento da PSE em ambos os protocolos. O aumento da PSE local (percepção de esforço da musculatura ativa) ao longo das séries tem sido reportado na literatura (HARDEE et al., 2012). É possível que tal resposta esteja associada a diferentes fatores, como aumentos do $\mathrm{pH}$ sanguíneo e redução da fosfocreatina (LAGALLY et al., 2002), levando o sujeito a indicar maiores valores na escala de esforço.

Com relação à comparação da PSE local entre os protocolos TRAD e SS, não foram observadas diferenças entre as situações experimentais. Esse resultado contraria respostas observadas por Souza et al. (2017), no qual o protocolo SS promoveu maiores valores de PSE que o protocolo TRAD. Convém destacar, entretanto, que na pesquisa de Souza et al. (2017) o sistema SS aplicado (agonista-antagonista) foi organizado diferentemente do presente estudo (alternado por segmento). Adicionalmente, os autores não procuraram equiparar as pausas entre séries nos protocolos estudados, nem detalharam se a PSE foi mensurada como uma percepção corporal geral ou apenas nas musculaturas ativas durante os exercícios. Destaca-se ainda que outros estudos que coletaram 
A realização do sistema supersérie com diferentes segmentos corporais... Ricardo Reis Dinardi • Guilherme Augusto Coelho Oliveira, et al...

dados de PSE após 15 minutos da sessão de treinamento (PSE sessão) demonstraram também maiores valores na condição SS (WEAKELEY et al., 2017a; WEAKELEY et al., 2017b). Conforme mencionado, a literatura tem indicado que tanto a pausa entre séries (HARDEE et al., 2012) quanto o VTT (HISCOCK et al., 2016) impactaram na resposta da PSE local. Logo, em função dessas variáveis terem sido semelhantes entre as situações experimentais aqui testadas, talvez os protocolos tenham provocado níveis similares de esforço percebido nos músculos responsáveis pelos exercícios. Sugere-se que estudos futuros realizem medidas de PSE com diferentes métodos (geral, local e por sessão) ao se comparar sistemas de treinamento SS e TRAD na musculação

O uso de apenas um par de exercícios pode ser compreendido como uma limitação desta pesquisa. Neste sentido, o resultado encontrado não pode ser extrapolado para outras condições de treinamento, como a realização de vários exercícios em uma mesma sessão. Pesquisas futuras deverão analisar o impacto da utilização das SS em rotinas completas de treinamento. Salienta-se ainda a necessidade de próximas investigações analisarem o uso do sistema SS em exercícios multiarticulares (ex: supino e agachamento).

\section{Conclusão}

Os resultados do presente estudo demonstraram que protocolos TRAD e SS promovem respostas similares de desempenho (volume de treinamento) e de percepção de esforço. Sendo assim, a aplicação de SS torna-se uma alternativa eficiente para a redução do tempo de treinamento. 
A realização do sistema supersérie com diferentes segmentos corporais... Ricardo Reis Dinardi • Guilherme Augusto Coelho Oliveira, et al...

\section{Referências}

ACSM. American College of Sports Medicine Position Stand. Progression Models in Resistance Training for Healthy Adults.

Medicine and Science in Sports and Exercise, v. 41, n. 3, p. 687708. 2009.

ALCARAZ, P.; SÁNCHEZ-LORENTE, J.; BLAZEVICH, A. Physical performance and cardiovascular responses to an acute bout of heavy resistance circuit training versus traditional strength training. Journal of Strength and Conditioning Research, v. 22, n. 3, p. 667-671, 2008.

BALSAMO, S.; TIBANA, R.; NASCIMENTO, D.; FARIAS, G.; PETRUCCELLI, Z.; SANTANA, F.; MARTINS, O.; AGUIAR, F.; PEREIRA, G.; SOUZA, J.; PRESTES, J. Exercise order affects the total training volume and the ratings of perceived exertion in response to a super-set resistance training session. International Journal of General Medicine, v. 5, p. 123-127, 2012.

BIRD, S.; TARPENNING, K.; MARINO, F. Designing resistance training programmes to enhance muscular fitness: a review of the acute programme variables. Sports Medicine, v. 35, n. 10, p. 841-851, 2005.

BORG, G. Escalas de Borg para a dor e o esforço percebido. $1^{\text {a }}$ edição. São Paulo, Manole, 2000.

CORRÊA, M.; CARVALHO, E.; BORGES, E.; OLIVEIRA, E.; FARIAS, D. Efeitos do método pareado agonista-antagonista utilizando séries múltiplas sobre o desempenho de repetições máximas para membros inferiores. ConScientiae Saúde, São Paulo, v. 16, n. 4, p. 409-416, 2017.

DINIZ RC, MARTINS-COSTA HC, MACHADO SC, LIMA FV, CHAGAS $\mathrm{MH}$. Repetition duration influences ratings of perceived exertion. Perceptual and Motor Skills, v. 118, n. 1, p. 261-273, 2014 FLECK, S.; KRAEMER, W. Fundamentos do treinamento de força muscular. $4^{\circ}$ ed., Porto Alegre, Artmed, 2017. 
A realização do sistema supersérie com diferentes segmentos corporais... Ricardo Reis Dinardi • Guilherme Augusto Coelho Oliveira, et al...

Hardee JP, Lawrence MM, Utter AC, Triplett NT, Zwetsloot KA,

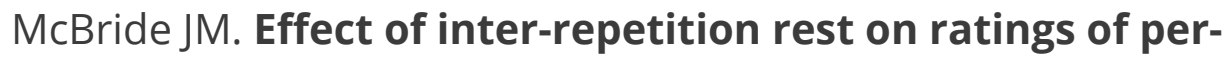
ceived exertion during multiple sets of the power clean. Eur J Appl Physiol. 2012 Aug;112(8):3141-7.

GEARHART, R.; GOSS, F.; LAGALLY, K.; JAKICIC, J.; GALLAGHER, J.; ROBERTSON, R. Standardized scaling procedures for rating perceived exertion during resistance exercise. Journal of Strength and Conditioning Research, v. 15, n. 3, p. 320-325, 2001.

HISCOCK, D.; DAWSON, B.; DONNELLY, C.; PEELING, P. Muscle activation, blood lactate, and perceived exertion responses to changing resistance training programming variables. European Journal of Sports Sciences, v. 16, n. 5, p. 536-544, 2016

KRAEMMER, W.; RATAMESS, N. Fundamentals of Resistance Training: Progression and Exercise Prescription. Medicine and Science in Sports and Exercise, v. 36, n. 4, p. 674-688, 2004.

LAGALLY, K.; ROBERTSON, R.; GALLAGHER, K.; GOSS, F.; JAKICIC, J.; LEPHART, S.; MCCAW, S.; GOODPASTER, B. Perceived exertion, electromyography, and blood lactate during acute bouts of resistance exercise. Medicine and Science in Sports and Exercise, $v$. 34, n. 3, p. 552-559, 2002

LIZ, C.; ANDRADE, A. Análise qualitativa dos motivos de adesão e desistência da musculação em academias. Revista Brasileira de Ciências do Esporte, Brasília, v. 38, n. 3, p. 267-274, 2016.

MAIA, M.; WILLARDSON, J.; PAZ, G.; MIRANDA, H. Effects of different rest intervals between antagonists paired sets on repetition performance and muscle activation. Journal of Strength and Conditioning Research, v. 28, n. 9, p. 2529-2535, 2014

MÁRQUEZ, G.; ROMERO-ARENAS, S.; MARÍN-PAGÁN, C.; VERAIBAÑEZ, A.; DEL OLMO, M.; TAUBE, W. Peripheral and central fatigue after high intensity resistance circuit training. Muscle Nerve, v. 56, n. 1, p. 152-159, 2017.

MONTEIRO, E.; MELO, L.; GARCIA, T.; PAZ, G.; SALLES, B.; DIAS, I.; MIRANDA, H. Efeito da ordem de execução de séries alternadas 
A realização do sistema supersérie com diferentes segmentos corporais... Ricardo Reis Dinardi • Guilherme Augusto Coelho Oliveira, et al...

por segmento comparadas a séries tradicionais sobre o desempenho de repetições máximas em diferentes segmentos corporais. Revista Brasileira de Prescrição e Fisiologia do Exercício, Maranhão, v. 55, n. 9, p. 519-525, 2015.

NACLERIO, F.; CHAPMAN, M.; LARUMBE-ZABALA, E. Use of the rate of perceived exertion scales in resistance training: A comment on Mayo, Iglesias-Soler, And Fernández-Del-Olmo ( 2014 ). Perceptual and Motor Skills, v. 121, n. 2, p. 490-493, 2015. NASSER, I.; FREITAS, D.;2, PAZ, G.; WILLARDSON, J.; MIRANDA, H. Volume load and efficiency with different strength training methods. Archivos de Medicina del Deporte, v. 36, n. 3, 2019. PAZ, G.; MAIA, M.; LIMA, V.; MIRANDA, H. Efeito do método agonista-antagonista comparado ao tradicional no volume e ativação muscular. Revista Brasileira de Atividade Física e Saúde, Florianópolis, v. 19, n. 1, p. 54-63, 2014.

RHEA, M.; ALVAR, B.; BURKETT, L.; BALL, SD. A meta-analysis to determine the dose response for strength development. Medicine and Science in Sports and Exercise, v. 35, n. 3, p. 456-564, 2003. ROBBINS, D.; YOUNG, W.; BEHM, D.; PAYNE, W. Effects of agonist-antagonist complex resistance training on upper body strength and power development. Journal of Sports Science, v. 27, n.14, p. 1617-1625, 2009.

ROBBINS, D.; YOUNG, W.; BEHM, D.; PAYNE, W.; KLIMSTRA, M. Physical performance and electromyographic responses and efficiency. Journal of Strength and Conditioning Research, v. 24, n. 5, p. 1237-1245, 2010a.

ROBBINS, D.; YOUNG, W.; BEHM, D.; PAYNE, W. Agonist-antagonist paired set resistance training: a brief review. Journal of Strength and Conditioning Research, v. 24, n. 10, p. 2873-2882, 2010 b.

SCHOENFELD, B.; OGBORN, D.; KRIEGER, J. Dose-response relationship between weekly resistance training volume and increases in muscle mass: A systematic review and meta-analysis. Journal of Sports Science, v. 35, n. 11, p. 1073-1082, 2017. 
A realização do sistema supersérie com diferentes segmentos corporais...

Ricardo Reis Dinardi • Guilherme Augusto Coelho Oliveira, et al...

SENNA, G.; BRITTO, R.; GOMES, T.; BASTOS, A.; NOVAES, J. Influência de dois diferentes tempos de intervalos entre exercícios e métodos de treinamento no desempenho da força. Revista Brasileira de Prescrição e Fisiologia do Exercício, Maranhão, v. 24, n. 4, p. 593-599, 2010.

SOUZA, J.; PAZ, G.; MIRANDA, H. Blood lactate concentration and strength performance between agonist-antagonist paired set, superset and traditional set training. Archivos de Medicina del Deporte, v. 34, n. 3, p. 145-150, 2017.

SUCHOMEL, T.; NIMPHIUS, S.; BELLON, C.; STONE, M. The Importance of Muscular Strength: Training Considerations. Sports Medicine, v. 48, n. 4, p. 765-785, 2018.

TIGGEMANN, C.; PINTO, R.; KRUEL, L. A percepção de esforço no treinamento de força. Revista Brasileira de Medicina do Esporte, São Paulo, v. 16, n. 4, p. 301-309, 2010.

WEAKLEY, J.; TILL, K.; READ, D.; ROE, G.; DARRALL-JONES, J.; PHIBBS, P.; JONES, B. The effects of traditional, superset, and tri-set resistance training structures on perceived intensity and physiological responses. European Journal of Applied Physiology, v. 117, n. 9, p. 1877-1889, 2017a.

WEAKLEY, J.; TILL, K.; READ, D.; PHIBBS, P.; ROE, G.; DARRALLJONES, J.; JONES, $B$ The effects of superset configuration on kinetic, kinematic, and perceived exertion in the barbell bench press. Journal of Strength and Conditioning Research, 2017b. No prelo.

WILLARDSON, J. A brief review: factors affecting the length of the rest interval between resistance exercise sets. Journal of Strength and Conditioning Research, v. 20, n. 4, p. 978-984, 2006. 
A realização do sistema supersérie com diferentes segmentos corporais...

Ricardo Reis Dinardi • Guilherme Augusto Coelho Oliveira, et al...

\section{Aprovação de comitê de ética em pesquisa}

Pesquisa aprovada pelo Comitê de Ética da Pontifícia Universidade Católica de Minas Gerais. Título: Efeito agudo do protocolo "super-série" no desempenho físico e percepção de esforço de praticantes de musculação.

\section{Publisher}

Universidade Federal de Goiás. Faculdade de Educação Física e Dança. Publicação no Portal de Periódicos UFG. As ideias expressadas neste artigo são de responsabilidade de seus autores, não representando, necessariamente, a opinião dos editores ou da universidade. 